\title{
Ranitidine bismuth citrate with clarithromycin for the treatment of duodenal ulcer
}

\author{
K D Bardhan, C Dallaire, H Eisold, A E Duggan
}

\begin{abstract}
BackgroundlAims-To investigate the effect of the new Helicobacter pylori eradication regimen, ranitidine bismuth citrate (RBC) and clarithromycin (CLAR) dual therapy, on duodenal ulcer healing and absence of ulcer recurrence during 24 weeks follow up (overall success).

Methods-Two hundred and thirty two $H$ pylori positive patients with active duodenal ulcer received four weeks treatment with RBC $400 \mathrm{mg}$ twice daily alone (RBC400) $(n=82)$, or RBC 400 or $800 \mathrm{mg}$ twice daily co-prescribed with clarithromycin $250 \mathrm{mg}$ four times daily for 14 days, followed by 14 days of RBC $400 \mathrm{mg}$ twice daily alone (RBC400+CLAR and RBC $800+C L A R$, respectively, $n=75$ for each). Results-The co-prescription regimens gave high $H$ pylori eradication rates determined using two tests (CLOtest and ${ }^{13} \mathrm{C}$-urea breath test) for the presence of the organism. These rates were $92 \%$ and $81 \%$ for RBC400+CLAR $(n=62)$ and RBC800+CLAR $(n=63)$ respectively, compared with $2 \%$ for RBC400 $(n=66)$ $(p<0.001)$. With respect to overall success as estimated by life table analysis, RBC400+CLAR (89\%) and RBC800+ CLAR $(87 \%)$ were significantly more effective than RBC400 alone (51\%) $(p<0.001)$. All regimens were safe and well tolerated. Trough plasma bismuth concentrations at week 4 were low (treatment medians less than $6.6 \mathrm{ng}$ bismuth/ml).

Conclusions-Ranitidine bismuth citrate is a well tolerated and efficacious ulcer healing drug which, when co-prescribed with clarithromycin, affords effective $H$ pylori eradication therapy and prevents ulcer relapse in most patients with duodenal ulcer.

(Gut 1997; 41: 181-186)
\end{abstract}

Keywords: ranitidine bismuth citrate; duodenal ulcer; Helicobacter pylori eradication

H Eisold

GlaxoWellcome

Research and

Development, UK

A E Duggan

Correspondence to: Dr K D Bardhan, Rotherham General Hospitals NHS Trust, Moorgate Road, Oakwood, Rotherham, South Yorkshire S60 2UD, UK.

Accepted for publication 20 February 1997 Ranitidine bismuth citrate (GR122311X, Pylorid $^{\mathrm{TM}}$, Tritec $\left.{ }^{\mathbb{B}}\right)$ is a novel salt of ranitidine (cation) with bismuth and citrate (anion). It possesses both the antisecretory activity of ranitidine and the mucosal protective and antiHelicobacter pylori effects of certain bismuth salts. ${ }^{1}$ The drug is as effective as ranitidine hydrochloride in healing duodenal ulcer ${ }^{2}{ }^{3}$ and more effective in healing gastric ulcer. ${ }^{4}$ When evaluated in a pilot study in co-prescription with the macrolide antibiotic clarithromycin, ranitidine bismuth citrate greatly enhanced the $H$ pylori eradication rate found previously with clarithromycin monotherapy. ${ }^{56}$ Furthermore, synergy between ranitidine bismuth citrate and clarithromycin for inhibitory and bactericidal activity against $H$ pylori has been shown in vitro in chequerboard and time/kill experiments and in vivo in mice.

In view of these promising results, we have conducted a controlled clinical trial using this dual therapy at different doses of ranitidine bismuth citrate in a larger population to determine the efficacy and safety of the regimen in the treatment of duodenal ulcer.

\section{Methods}

This multinational clinical trial was conducted in Belgium, Canada, Denmark, Germany, Iceland, Spain, Sweden, Switzerland, and the UK. It was approved by the appropriate local ethics committees and, where required, the national regulatory authorities.

\section{PATIENTS}

Patients aged $18-80$ years with endoscopically proven active duodenal ulcer $(5-20 \mathrm{~mm})$ and $H$ pylori infection detected initially by a positive CLOtest $^{\mathrm{TM}}$ (CLOtest is a registered trademark of Delta West Ltd, Perth, Australia) on either two gastric antral or two gastric corpus biopsy specimens (one pair of biopsy specimens per slide), and who gave informed written consent, were included. If $H$ pylori infection was subsequently not confirmed by a positive ${ }^{13} \mathrm{C}$-urea breath test (UBT) (positive result $=$ excess delta ${ }^{13} \mathrm{CO}_{2}>5$ per mil) the patient was to be withdrawn from the trial. The following patients were excluded: those who had concurrent erosive oesophagitis, pyloric ulcers, gastric ulcers, or previous gastric surgery; those with a bleeding ulcer; those who had in the previous 30 days taken drugs which affect $H$ pylori; and those likely to need treatment with ulcerogenic drugs. Other exclusions were for safety reasons.

DESIGN

In a randomised, double blind, double dummy, eradication of $H$ pylori infection: ranitidine bismuth citrate (RBC) $400 \mathrm{mg}$ twice daily (RBC400) plus placebo clarithromycin (CLAR); RBC $400 \mathrm{mg}$ twice daily coprescribed with clarithromycin $250 \mathrm{mg}$ four times daily (RBC400+CLAR); or RBC $800 \mathrm{mg}$ twice daily co-prescribed with clarithromycin $250 \mathrm{mg}$ four times daily (RBC800+CLAR). Doses of ranitidine bis- 
muth citrate were taken on an empty stomach; the clarithromycin was taken after food. All patients then received a further 14 days of ranitidine bismuth citrate $400 \mathrm{mg}$ twice daily for ulcer healing and symptom relief (ranitidine bismuth citrate $400 \mathrm{mg}$ and ranitidine hydrochloride $150 \mathrm{mg}$ contain equivalent amounts of ranitidine base).

No concurrent therapy with antiulcer treatments, antacids (except for low doses for up to one week during the follow up phase), antibiotics or antibacterials, gold, non-steroidal anti-inflammatory drugs, and other potentially ulcerogenic drugs or bismuth containing compounds was permitted. During treatment patients were assessed at two weeks for adverse events and compliance, and at four weeks by endoscopy, CLOtests on two antral and two corpus biopsies, and UBT to assess ulcer healing and $H$ pylori status. Patients with unhealed ulcers were withdrawn, treated with other drugs at the physician's discretion and reviewed 14-21 days later by safety assessment and UBT.

At prestudy and each visit during the treatment phase the investigator questionned the patient and noted the presence and severity of ulcer related symptoms (including ulcer pain) over the preceding two days. Severity of symptoms was graded as none, mild, moderate, or severe in relation to extent of interference with daily activities.

Patients with healed ulcers (defined as complete re-epithelisation of the ulcer and continuity of the duodenal mucosa) at four weeks were followed up untreated for a further 24 weeks. Patients underwent endoscopy and UBT at four, 12, and 24 weeks after cessation of treatment, or underwent endoscopy earlier if persistent symptoms developed. Breath test method was as for the variant of the European Standard Protocol, ${ }^{8}$ which uses a single sample collected by straw 30 minutes post-ingestion of ${ }^{13} \mathrm{C}$-urea. Patients with ulcer recurrence were withdrawn from the study and treated at the physician's discretion.

SAFETY ASSESSMENTS

At each visit patients were questionned about adverse events which were recorded and their relationship to study medication was assessed by the investigator. Blood samples were taken from all patients prestudy, at week 2 , and at the end of treatment (median 11.5 hours after the last treatment dose) to determine trough plasma bismuth concentrations. Bismuth was determined by inductively coupled plasma mass spectroscopy ${ }^{9}$ at the Elemental Research Institute, Canada (limit of detection $0.2 \mathrm{ng} /$ $\mathrm{ml})$.

\section{STATISTICAL METHODS}

The study was designed to have $80 \%$ power to detect simultaneously a $30 \%$ difference in overall success between RBC $400 \mathrm{mg}$ twice daily alone and both of the co-prescription therapies (assuming that two sided tests at the $5 \%$ significance level were used). A total of 56 patients in each treatment group was needed; this was increased by $20 \%$ to compensate for drop outs during the 24 week follow up period.

Reported analyses relating to ulcer status include all available data from all patients with duodenal ulcer who were randomised to receive treatment. Analyses relating to $H$ pylori status exclude only those patients with unconfirmed $H$ pylori status prestudy, or for whom data were unavailable at a given predefined time interval.

\section{Primary efficacy parameter}

This was the percentage of patients who were healed at end of treatment and who then remained ulcer free during 24 weeks of follow up (overall success) - that is, a combination of the two clinical outcomes required for successful duodenal ulcer disease management. $\mathrm{Cu}-$ mulative overall success rates were determined by Kaplan-Meier life table analysis (using the observed ulcer healing rate and the proportion of patients who were ulcer free at each subsequent visit) and treatments were compared using log rank tests. ${ }^{10}$

\section{Secondary efficacy parameters}

These were ulcer healing, ulcer relapse, and $H$ pylori eradication rates.

Intention to treat and observed ulcer healing rates were calculated by inclusion of all randomised patients (those not assessed were assumed to have unhealed ulcers), and only those who underwent endoscopy after four weeks of treatment, respectively. The healing rates were compared using Mantel-Haenzsel $\chi^{2}$ statistical tests.

Ulcer relapse rates for all patients with healed ulcers (who were not withdrawn at the end of treatment) were determined from the number of observed relapses by week 24 of follow up (best case rate) and by life table analysis (worst case).

Assessment of $H$ pylori status

Only those patients who met the strict criteria of having both a positive CLOtest and UBT at entry were eligible for analysis of $H$ pylori eradication. Collection of data on $H$ pylori status was scheduled at a further four visits. Calculation of eradication rates was primarily based on data from the visit 28 days posttreatment, but in the absence of evaluable data at that time, an earlier positive result or later negative results were then utilised.

\section{$H$ pylori eradication}

The criteria used for successful eradication (two negative results) were more exacting than for failed treatment (one positive result). $H$ pylori eradication was deemed successful if the patient was simultaneously found to be negative by both CLOtest and UBT at least 28 days after the end of treatment and with no prior positive post-treatment test. Patients were classified into two categories on the basis of their test data. Eligible patients were those with either two evaluable negative tests simultaneously conducted at least 28 days after the end of treatment, or with one positive evaluable test between the end of treatment and the 12 week 
TABLE 1 Demography of patients randomised to therapy

\begin{tabular}{llll}
\hline Treatment & RBC400 & RBC400+CLAR & RBC800+CLAR \\
\hline Number of patients & 82 & 75 & 75 \\
Age (mean (SD) years) & $49(13)$ & $49(14)$ & $52(12)$ \\
Male/female (\%) & $78 / 22$ & $57 / 43$ & $76 / 24$ \\
Smokers (\%) & 42 & 45 & 48 \\
Drinkers (\%) & 69 & 57 & 57 \\
Previous ulcer history (\%) & 77 & 69 & 67 \\
Ulcer pain at entry (\%) & 94 & 95 & 93 \\
Prestudy positive for H pylori (\%) & 90 & 92 & 96 \\
\hline
\end{tabular}

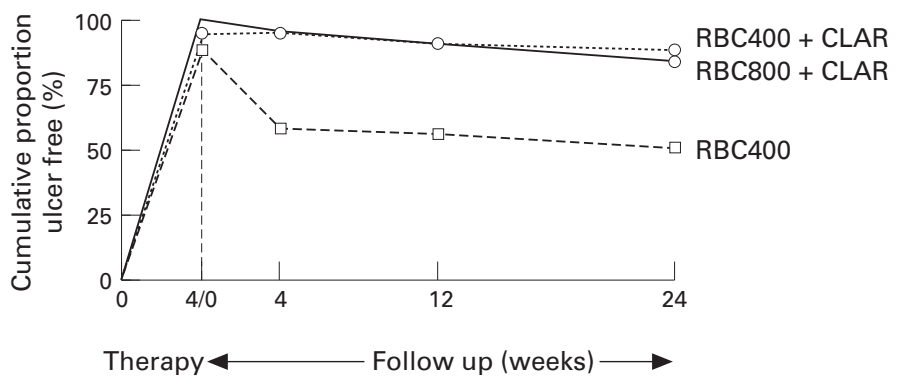

$\begin{array}{rlllll}\text { RBC400 + CLAR } & 75 & 70 & 61 & 57 & 53 \\ \text { RBC800 + CLAR } & 75 & 72 & 65 & 59 & 53 \\ \text { RBC400 } & 82 & 76 & 60 & 32 & 29\end{array}$

Figure 1: Cumulative overall success rates-life table analysis. Numbers at the bottom of the graph are of patients assessed at each time point in the three different treatment groups. Overall success, the primary end point, combines in a single value the proportions of patients whose ulcer healed and remained so at the end of 24 weeks' follow up.

post-treatment visit, thus including patients with a positive UBT who were withdrawn early or had an unhealed ulcer. (The CLOtest was considered evaluable if there was at least one positive biopsy result, or if there were four biopsy specimens taken and all were negative. A combination of negative and missing results was classified as an unevaluable CLOtest.) Ineligible patients were those whose results might be falsely negative (for example, tests conducted less than 28 days post-treatment through premature withdrawal or misscheduling, or a combination of the failure of one test with a second negative test). Two $H$ pylori eradication rates have been calculated:

\section{Intention to treat eradication rate}

All randomised patients are included in the denominator and ineligible patients are thereby assumed to be treatment failures. This is the "worst case" and eradication might be underestimated

Observed eradication rate

In contrast to the above this may give a more realistic assessment as only eligible patients (for example, one positive or two negative test results) are included in the denominator. The effects of treatment on observed $H$ pylori eradication were compared using stratified MantelHaenzsel $\chi^{2}$ tests.

RECRUDESCENCE OF H PYLORI

This was assessed from the later development of a positive CLOtest or UBT when both tests had previously been negative at least 28 days post-treatment.

\section{Results}

PATIENTS

A total of 232 patients with duodenal ulcer were randomised to treatment. The groups of patients receiving each treatment were well matched with regard to baseline measures (other than sex) (table 1). The presence of $H$ pylori infection at prestudy was not confirmed by UBT for 17 patients, and 11 were withdrawn from the study; all were excluded from all analyses involving eradication of $H$ pylori. Eighty nine patients withdrew before completion of the 28 week study period: 52 from the RBC400 group, 18 from the RBC400+CLAR group, and 19 from the RBC800+CLAR group.

OVERALL SUCCESS RATE

The proportion of patients whose ulcers were healed and who were still in remission 24 weeks after the end of treatment was estimated by life table analysis to be as follows: RBC400, $51 \%$; RBC400+CLAR, 89\%; and RBC800+ CLAR, $87 \%$ (fig 1). The cumulative overall success rates were clinically and statistically significantly higher with RBC400+CLAR and RBC800+CLAR than with RBC400 $(\mathrm{p}<0.001)$. The results were not altered if those patients who were not confirmed to be $H$ pylori positive prestudy were excluded from the analysis. In the second co-prescription regimen the higher $800 \mathrm{mg}$ dose of ranitidine bismuth citrate did not increase the benefit of the $400 \mathrm{mg}$ dose.

HEALING

The intention to treat healing rates after four weeks of treatment were high for all three regimens with a statistically significant difference between RBC400 and RBC800+CLAR $(\mathrm{p}=0.041)($ table 2). Observed healing rates were $90 \%, 96 \%$, and $97 \%$, respectively, for RBC400, RBC400+CLAR, and RBC800+ CLAR; none of these differences were statistically significant.

RELAPSE ON FOLLOW UP

Informal comparisons of cumulative ulcer relapse rates at the end of 24 weeks showed those for RBC400+CLAR (7\%) and RBC $800+$ CLAR $(10 \%)$ to be significantly lower than that for RBC400 alone $(44 \%)(\mathrm{p}<0.001$ for both comparisons) (table 2).

HELICOBACTER PYLORI

At least $90 \%$ of patients in all groups were $H$ pylori positive on both CLOtest and UBT before treatment (table 1).

In the analysis of all patients evaluable for assessment of observed $H$ pylori eradication, both RBC400+CLAR and RBC800+CLAR were significantly more effective than RBC400 in the eradication of $H$ pylori $(92 \%, 81 \%$, and $2 \%$, respectively) and by intention to treat analysis $(83 \%, 71 \%$, and $1 \%$, respectively).

Of patients who were $H$ pylori positive prestudy, eight, seven, and nine patients in the RBC400, RBC400+CLAR, and RBC800+ CLAR groups, respectively, were excluded 
TABLE 2 Ulcer healing, relapse, overall success, and eradication of H pylori

\begin{tabular}{|c|c|c|c|c|c|c|c|c|c|}
\hline & \multicolumn{3}{|c|}{$R B C 400$} & \multicolumn{3}{|c|}{$R B C 400+C L A R$} & \multicolumn{3}{|c|}{$R B C 800+C L A R$} \\
\hline & $\%$ & $95 \% C I$ & $n$ & $\%$ & $95 \% C I$ & $n$ & $\%$ & $95 \% C I$ & $n$ \\
\hline \multicolumn{10}{|l|}{ Ulcer healing week 4} \\
\hline Intention to treat & 83 & $73-90$ & $68 / 82$ & 89 & $80-95$ & $67 / 75$ & $93^{\star \star}$ & $85-98$ & $70 / 75$ \\
\hline Observed & 90 & $80-95$ & $68 / 76$ & 96 & $88-99$ & $67 / 70$ & 97 & $90-100$ & $70 / 72$ \\
\hline \multicolumn{10}{|l|}{ Ulcer relapset } \\
\hline Life table analysis week 24 relapse & 44 & $30-57$ & 63 & $7^{\star}$ & $0-14$ & 64 & $10^{\star}$ & $2-18$ & 69 \\
\hline Intention to treat week 24 relapse & 40 & $28-53$ & $25 / 63$ & $6^{\star}$ & $2-15$ & $4 / 64$ & $9^{\star}$ & $3-18$ & $6 / 69$ \\
\hline \multicolumn{10}{|l|}{ Cumulative overall success week 24} \\
\hline Life table analysis & 51 & $38-63$ & 82 & $89^{\star}$ & $81-97$ & 75 & $87^{\star}$ & $79-96$ & 75 \\
\hline \multicolumn{10}{|l|}{ H pylori eradication } \\
\hline Observed rate ${ }^{1}$ & 2 & $0-8$ & $1 / 66$ & $92^{\star}$ & $82-97$ & $57 / 62$ & $81^{\star}$ & $69-90$ & $51 / 63$ \\
\hline Intention to treat eradication rate ${ }^{2}$ & 1 & $0-7$ & $1 / 74$ & $83^{\star}$ & $72-91$ & $57 / 69$ & $71^{\star}$ & $59-81$ & $51 / 72$ \\
\hline \multicolumn{10}{|l|}{$\begin{array}{l}\text { Relapse in relation to } H \text { pylori } \\
\text { eradication }\end{array}$} \\
\hline \multicolumn{10}{|l|}{ Duodenal ulcer relapse when: } \\
\hline H pylori eradicated ${ }^{3}$ & & & $0 / 1$ & & & $2 / 57$ & & & $0 / 51$ \\
\hline H pylori not eradicated ${ }^{3}$ & & & $22 / 56$ & & & $2 / 5$ & & & $5 / 10$ \\
\hline Eradication status unevaluable ${ }^{4}$ & & & $1 / 3$ & & & $0 / 1$ & & & $1 / 6$ \\
\hline$H$ pylori at prestudy not confirmed ${ }^{4}$ & & & $2 / 3$ & & & $0 / 1$ & & & $0 / 2$ \\
\hline \multicolumn{10}{|l|}{ H pylori status at relapse } \\
\hline H pylori positive & & & $23^{5} / 23$ & & & $3^{5} / 4$ & & & $5 / 6^{6}$ \\
\hline Recrudescence/reinfection ${ }^{7}$ & & 100 & $1 / 1$ & & 7 & $4 / 56$ & & 6 & $3 / 48$ \\
\hline
\end{tabular}

${ }^{\star} \mathrm{p}<0.001 v$ RBC400+placebo; ${ }^{\star \star} \mathrm{p}<0.041 v$ RBC400+placebo.

tInformal comparison only performed as patients not re-randomised post-treatment and differential healing rates could introduce bias.

Method of calculation

All patients included in the eradication analyses were positive prestudy by both CLOtest and UBT

Post-treatment $H$ pylori regative is defined as both CLOtest and UBT negative at least 28 days post-treatment, and $H$ pylori positive is defined as at least one positive result.

${ }^{1}$ Observed eradication rate is the number of patients $H$ pylori negative at least 28 days after the end of treatment divided by the number of patients with both an evaluable negative CLOtest and UBT at a visit at least 28 days after the end of treatment, or a single positive test at any time between the end of treatment and before the week 12 visit.

${ }^{2}$ Intention to treat eradication rate is as above but patients with unevaluable data were assumed to have been unsuccessfully treated and are added to the denominator.

${ }^{3}$ Analysis includes all healed patients who were defined as $H$ pylori positive at prestudy, entered follow up phase, and for whom $H$ pylori eradication status was available.

${ }^{4}$ Patients with unconfirmed $H$ pylori status at prestudy or 28 days post-treatment, who were not withdrawn and for whom endoscopic assessments were available.

${ }^{5}$ One patient per group with ulcer relapse was unevaluable for eradication assessment, but at time of relapse was positive.

${ }^{6}$ The negative patient was classified as positive at the eradication assessment but then negative at relapse at week $12-$ no concomitant medication was recorded to explain this anomalous result.

${ }^{7}$ Reinfection/recrudescence rate is the number of patients defined as $H$ pylori negative at least 28 days after the end of treatment who have at least one positive test at a later week 12 or 24 visit divided by the number of patients defined as $H$ pylori eradicated with at least one later evaluable visit (up to week 24).

from the observed eradication analysis due to failure to return or lack of data for another reason (see table 3).

Failure of co-prescription treatment to eradicate $H$ pylori could not be explained in most of the 17 cases; one of these patients took less than $80 \%$ of the study medication, but the remainder had plasma bismuth concentrations indicative that medication had been taken. (Conversely three "non-compliant" patients had successful eradication.) Prestudy macrolide susceptibility was not examined.

TABLE 3 Reasons for exclusion from observed eradication analysis four to 24 weeks post-treatment

\begin{tabular}{llll}
\hline & $\begin{array}{l}R B C 400 \\
(n)\end{array}$ & $\begin{array}{l}R B C 400+C L A R \\
(n)\end{array}$ & $\begin{array}{l}R B C 800+C L A R \\
(n)\end{array}$ \\
\hline $\begin{array}{l}\text { Patients without a positive prestudy } \\
\text { UBT }\end{array}$ & 8 & 6 & 3 \\
$\begin{array}{c}\text { Patients withdrawn due to adverse event } \\
\text { prior to eradication assessment }\end{array}$ & 3 & 3 & 2 \\
$\begin{array}{l}\text { Patients withdrawn due to unhealed } \\
\text { ulcer }\end{array}$ & 2 & 3 & 1 \\
$\begin{array}{l}\text { Patients failed to return/opted to } \\
\text { discontinue }\end{array}$ & 2 & 1 & 5 \\
$\begin{array}{c}\text { Patients with only one evaluable } \\
\text { negative test or two negative tests but } \\
\text { earlier than day 28 }\end{array}$ & 1 & 0 & 1 \\
$\begin{array}{l}\text { Patients evaluable for eradication week } 4 \\
\text { to week 24 }\end{array}$ & 66 & 62 & 63 \\
\hline
\end{tabular}

RELATIONSHIP BETWEEN H PYLORI STATUS AND ULCER RELAPSE

Within each treatment group ulcer relapse occurred in proportionally less patients in whom $H$ pylori was eradicated than in those in whom it was not (table 2). Thirty one patients (of the 180 who were healed, entered the follow up phase, and were assessable for $H$ pylori eradication) had an ulcer relapse in this study. Only two of these were $H$ pylori negative at least 28 days after the end of treatment (table 2). Ulcer relapse data are also presented in table 2 for patients in whom either $H$ pylori status prestudy or at the eradication assessment was unconfirmed, but who failed to be withdrawn.

Forty one per cent $(29 / 71)$ of patients who had unsuccessful $H$ pylori eradication experienced an ulcer relapse, and at the time of relapse 31 of the 33 patients who relapsed (including two who were unevaluable at the eradication assessment) were $H$ pylori positive (table 2). Numbers are small, but ulcer relapse appears to occur later in the co-prescription groups than in the RBC400 group, perhaps suggesting a more prolonged suppression of $H$ pylori.

Of the 180 patients assessable for $H$ pylori eradication, 109 were deemed to have successful $H$ pylori eradication; of these one subsequently had an ulcer relapse with recrudes- 
TABLE 4 Patients experiencing the most common adverse events during the treatment phase

\begin{tabular}{|c|c|c|c|c|c|c|}
\hline & \multicolumn{2}{|c|}{$R B C 400$} & \multicolumn{2}{|c|}{$R B C 400+C L A R$} & \multicolumn{2}{|c|}{$R B C 800+C L A R$} \\
\hline & $\%$ & $n$ & $\%$ & $n$ & $\%$ & $n$ \\
\hline Safety population denominator & & 82 & & 75 & & 75 \\
\hline Patients with any adverse event ${ }^{1}$ & 29 & 24 & 28 & 21 & 25 & 19 \\
\hline Patients with drug related events ${ }^{1}$ & 18 & 15 & 17 & 13 & 16 & 12 \\
\hline $\begin{array}{l}\text { Patients withdrawn due to an } \\
\text { adverse event during treatment }\end{array}$ & 2 & 2 & 4 & 3 & 1 & 1 \\
\hline \multicolumn{7}{|l|}{ Most common': } \\
\hline Headache & 9 & 7 & 8 & 6 & 3 & 2 \\
\hline Diarrhoea & 4 & 3 & 5 & 4 & 7 & 5 \\
\hline Dark stools & 87 & 71 & 84 & 63 & 92 & 69 \\
\hline Black tongue & 6 & 5 & 12 & 9 & 15 & 11 \\
\hline
\end{tabular}

${ }^{1}$ Excludes dark stool (unless suspected melaena) or black tongue.

${ }^{2}$ Most common was defined as $\geqslant 5 \%$ in any treatment group.

cence of infection and another had ulcer relapse without evidence of recrudescence. One hundred and five of the successfully treated patients had a later visit at which recrudescence (or reinfection-these were indistinguishable as no strain typing was performed) could be assessed. Eight per cent of patients appeared to experience recrudescence, though seven of these eight patients remained ulcer free during the follow up period and the possibility of a single false positive test could not be excluded for five of these patients. None of the four patients who were unevaluable for recrudescence had an ulcer relapse.

PAIN RELIEF

The proportions of patients with ulcer pain at entry were similar for the three treatment groups: $94 \%$ for RBC400, $95 \%$ for RBC400+CLAR, and $93 \%$ for RBC800+ CLAR. Severity of pain was distributed similarly for each treatment group. All three regimens gave good symptom relief after four weeks of treatment: $79 \%$ of patients were pain free following RBC400 ( $n=77), 84 \%$ after RBC400+CLAR $(n=70)$, and $92 \%$ after RBC800+CLAR ( $n=72)$; the differences were not statistically significant.

SAFETY

All patients who were randomised to treatment took at least one dose of medication and have been included in the assessment of safety. The proportions of patients who experienced any events, whether judged treatment related or not by the investigator, were similar in all patient groups, as was the incidence of individual adverse events. Withdrawals due to adverse events during treatment were few and similar in the different groups (table 4). Observed darkening of stools during treatment and less frequently, black tongue, were noted in all treatment groups.

One patient reported a serious adverse event during treatment and was later withdrawn. The patient, who received RBC400+CLAR, had a urinary tract infection and renal colic which occurred on the twenty fourth day of treatment. This was not considered to be drug related.

The medians (and 95th centiles) for plasma bismuth concentrations at approximately 11.5 hours after the last dose of the four weeks of treatment were: 2.0 (7.7), 5.3 (21.1), and 6.5
(36.5) ng/ml after RBC400, RBC400+CLAR, and RBC800+CLAR, respectively.

\section{Discussion}

This blinded, controlled study showed that dual therapy with the new compound ranitidine bismuth citrate plus clarithromycin was both a well tolerated and very effective treatment, as judged by high overall success in the management of duodenal ulcer, which in turn was related to the high rates of $H$ pylori eradication achieved. Our results are supported by five other multicentre clinical trials which also found this dual therapy to be highly effective in the eradication of $H$ pylori. ${ }^{11-15}$

Traditional bismuth based dual therapy is less effective than bismuth based triple therapy which gives eradication rates of $73-94 \%,{ }^{16}$ but at the price of frequent side effects. Ranitidine based triple therapy is also very effective, giving $89 \%$ eradication. ${ }^{17}$ However, by using ranitidine bismuth citrate plus clarithromycin dual therapy we have achieved similar high levels of efficacy $(92 \%$ eradication) with minor side effects.

As expected with an $H$ pylori eradication rate of $92 \%$, a high proportion (89\%) of the patients who were randomised to RBC400+CLAR had their ulcers healed and remained ulcer free for 24 weeks. Eradication after RBC400 alone was poor $(2 \%)$ and only $51 \%$ of patients receiving this treatment had both ulcers healed and remained ulcer free at six months. This is in contrast to earlier claims that bismuth monotherapy results in significantly lower duodenal ulcer relapse than does $\mathrm{H}_{2}$ receptor antagonist therapy. ${ }^{18-20}$

Efficacy is expressed in terms of the clinical end point overall success, providing a single integrated measure that combines both ulcer healing and ulcer free remission for 24 weeks. This end point was selected in preference to ulcer relapse as patients were not rerandomised after healing, and thus a treatment effect could introduce bias after the initial randomisation process. Cumulative (worst case) relapse rates (life table analysis) can be calculated and the rate of $7 \%$ at 24 weeks after completion of the dual therapy treatment suggests that relapse rates are at the lower end of the range of those observed after triple therapies including bismuth (0-21\% after 12 months) and after antisecretory triple therapies $(9-41 \%) .^{21}$

The observed eradication rate reported here includes all evaluable test data from the 28 day to 24 week post-treatment period. A further 30 patients are included in the analysis compared with a previous analysis presented in abstract form, ${ }^{22}$ as the latter focused on data from the 28 day post-treatment visit only and excluded patients with less than two evaluable tests (although they may have had a single positive post-treatment test); such patients are included here.

Historical concerns about the potential toxicity of certain bismuth salts (subgallate and subnitrate) led us to pay particular attention to the determination of trough bismuth concentrations at two and four weeks of treatment. 
Plasma bismuth concentrations after dual therapy with RBC400+CLAR were higher than after RBC400 alone, but nevertheless in absolute terms were low and without clinical significance.

Dual therapy for the management of duodenal ulcer needs to be simple, consistently effective, and safe. Omeprazole plus amoxycillin gives widely varying $H$ pylori eradication results: from 0 to $92 \%$, the mean being $50-60 \%{ }^{21}$ Omeprazole plus clarithromycin is more effective (56-83\% eradication) ${ }^{21}$ than the combination with amoxycillin, but to date only ranitidine bismuth citrate plus clarithromycin meets all these requirements. It is concluded that two weeks of dual therapy with ranitidine bismuth citrate $400 \mathrm{mg}$ twice daily and clarithromycin $250 \mathrm{mg}$ four times daily, followed by a further two weeks of ranitidine bismuth citrate $400 \mathrm{mg}$ twice daily alone, provides ulcer healing and sustained remission from ulcer relapse together with corresponding high rates of $H$ pylori eradication.

Preliminary results were presented as an abstract at the Annual Meeting of the American Gastroenterological Association in San Diego, May 1995 (Gastroenterology 1995; 108: A53). The authors wish to thank John Forster, Claire Cutts, Larry Lacey, the country coordinators and monitors and the following principal investigators and their coworkers for their contribution to this study. Belgium: Dr P Barbier, Dr V Lamy, Professor G Ligny, Dr H Naessens, Dr J Pen, Dr J Toussaint, Dr J Van Isveldt, Dr L Van Waes; Canada Professor A P Archambault D R Dubé, Dr N Marcon, Dr T Ponich, Dr P Smith, Dr J Wright Denmark: Dr L Hendel, Dr P J Ranlov; Germany: Dr med D Berger, Dr med J C Boekhorst, Dr med H Brinkhoff, Dr med B Dirr, Dr med J Hagel, Dr med C Kupka, Dr med A Mares, Dr med W Peters-Haertel, Professor Dr med Rösch Dr med med W Peters-Haertel, Professor Dr med Rosch, Dr med G Scholz, Dr med E Schutz, Professor Dr med B Simon, Dr med H-J Taenzer; Iceland: Dr H Gudjonsson, Dr E Oddsson, Dr B Thjodleifsson; Spain: Dr J Ponce, Dr R Moreno; Sweden:

This study was sponsored by GlaxoWellcome Research and Development.

1 Stables R, Campbell CJ, Clayton NM, Clitherow JW, Grinham CJ, McColm AA, et al. Gastric antisecretory, mucosal protective, anti-pepsin and anti-Helicobacter properties of protective, anti-pepsin and anti-Helicobacter properties of ranitidin

2 Bardhan KD, Dekkers CPM, Lam SK, Nowak A, Schaffalitzky de Muckadell OB, Schütze K, et al. GR122311X (ranitidine bismuth citrate), a new drug for the treatment of duodenal ulcer. Aliment Pharmacol Ther 1995; 9: 497-506.

3 Forssell H, Nowak A, Tildesley G, Schütze K, Paré P, Sommer A, et al. Comparison of GR122311X (ranitidine bismuth citrate) with ranitidine hydrochloride for the treatment of duodenal ulcer [abstract]. Gut 1995; 37 (suppl 2): A41. 4 Bailey RJ, Marlicz K, Rosch W, Schaffalitzky de Muckadell
OB, Schulz T, Thjodleifsson B, et al. GR122311X (ranitidine bismuth citrate), a new drug for the treatment of gastric ulcer [abstract]. Gastroenterology 1995; 108: A51.

5 Wyeth JW, Pounder RE, Duggan AE, O'Morain CA, Schaufelberger HD, de Kosters EH, et al. The safety and efficacy of ranitidine bismuth citrate in combination with antibiotics for the eradication of Helicobacter pylori. Aliment Pharmacol Ther 1996; 10: 623-30.

6 Peterson WL, Graham DY, Marshall B, Blaser MJ, Genta $\mathrm{RM}$, Klein PD, et al. Clarithromycin as monotherapy for eradication of Helicobacter pylori: a randomized, doubleeradication of Helicobacter pylori: a randomized,
blind trial. Am 7 Gastroenterol 1993; 88: 1860-4.

7 McLaren A, McDowell SR, Bagshaw JA, McColm AA. The synergistic interaction between GR122311X and clarithromycin against Helicobacter [abstract]. Am $\mathcal{F}$ Gastroenterol 1994; 89: 1382

8 Logan RPH, Dill S, Bauer FE, Walker MM, Hirschl AM, Gummett PA, et al. The European ${ }^{13} \mathrm{C}$-urea breath test for the detection of Helicobacter pylori. Eur $\mathcal{f}$ Gastroenterol Hepatol 1991; 3: 915-21.

9 Tye DA, Tye DD, Brown R, Davis IM. Sensitive determination of bismuth concentration in human plasma, blood and urine using inductively coupled plasma mass spectrometry [abstract]. Pharm Res 1992; 9 (suppl 1): APQ1019.

10 Armitage P, Berry G. Chapter 14. In: Statistical methods in medical research. Oxford: Blackwell Scientific Publications, 1987.

11 Pounder RE, Wyeth JW, Duggan AE, Bailey RJ, Louw JA, Ohlin B. Ranitidine bismuth citrate with clarithromycin for the eradication of Helicobacter pylori and ulcer healing. Helicobacter 1997: in press.

12 Peterson WL, Ciociola AA, Sykes DL, McSorley DJ, Webb $\mathrm{DD}$, and the RBC H. pylori study group. Ranitidine bismuth citrate plus clarithromycin is effective for healing duodenal ulcers, eradicating $\mathrm{H}$. pylori, and reducing ulcer recurrence. Aliment Pharmacol Ther 1996; 10: 251-61.

13 Lanza F, Ciociola AA, Sykes D, Heath A, McSorley DJ, Webb DD. Ranitidine bismuth citrate plus clarithromycin is effective in eradication of $\mathrm{H}$. pylori, healing duodenal ulcers and preventing ulcer relapse [abstract]. Gastroenterology 1996; 110: A172.

14 Bardhan KD, Wurzer H, Marcelino M, Jahnsen J, Lotay N. High cure rates with ranitidine bismuth citrate (Pylorid) plus clarithromycin given twice daily [abstract]. Gut 1996; 39 (suppl 2): A36.

5 Axon ATR, Lancaster Smith MJ, Roopram PD. Ranitidine bismuth citrate and clarithromycin twice daily in the eradication of Helicobacter pylori. Aliment Pharmacol Ther 1997; 11: $81-8$.

16 Chiba N, Rao BV, Rademaker JW, Hunt RH. Meta-analysis of the efficacy of antibiotic therapy in eradicating

17 Hentschel E, Brandstatter G, Dragosics B, Hirschl AM, Nemec H, Schütze K, et al. Effect of ranitidine and amoxycillin plus metronidazole on the eradication of Helicobacter pylori and the recurrence of duodenal ulcer. $N$ Engl f Med 1993; 328: 308-12.

8 McLean AJ, McCarthy P, Dudley FJ. Cytoprotective agents and ulcer relapse. Med f Aust 1985; 142: S25-8.

19 Miller JP, Faragher EB. Relapse of duodenal ulcer: does it matter which drug is used in initial treatment? BMF 1986; 293: $1117-8$.

20 Dobrilla G, Vallaperta P, Amplatz S. Influence of ulcer healing agents on ulcer relapse after discontinuation of acute treatment: a pooled estimate of controlled clinical trials. Gut $1988 ; 29: 181-7$.

21 Dixon JS. Helicobacter pylori eradication: unravelling the facts. Scand F Gastroenterol 1995; 30 (suppl 212): 48-62

2 Bardhan KD, Dallaire C, Eisold H, Duggan AE. GR122311X (ranitidine bismuth citrate) with clarithromycin for the treatment of duodenal ulcer [abstract]. Gastroenterology 1995; 108 (4 part 2): A53. 